\title{
Evaluating Safer Conception Options for HIV-Serodiscordant Couples (HIV-Infected Female/HIV-Uninfected Male): A Closer Look at Vaginal Insemination
}

\author{
Okeoma Mmeje, ${ }^{1,2}$ Craig R. Cohen, ${ }^{1,2,3}$ and Deborah Cohan' \\ ${ }^{1}$ Department of Obstetrics, Gynecology \& Reproductive Sciences, University of California, San Francisco, San Francisco, \\ CA 94110, USA \\ ${ }^{2}$ UCSF Bixby Center for Global Reproductive Health, San Francisco, CA 94105, USA \\ ${ }^{3}$ Family AIDS Care and Education Services (FACES), Kenya
}

Correspondence should be addressed to Okeoma Mmeje, mmejeo@globalhealth.ucsf.edu

Received 21 February 2012; Accepted 6 July 2012

Academic Editor: Jean R. Anderson

Copyright ( $\odot 2012$ Okeoma Mmeje et al. This is an open access article distributed under the Creative Commons Attribution License, which permits unrestricted use, distribution, and reproduction in any medium, provided the original work is properly cited.

HIV serodiscordant couples represent at least half of all HIV-affected couples worldwide. Many of these couples have childbearing desires. Safer methods of conception may allow for pregnancy while minimizing the risk of sexual transmission of HIV. In serodiscordant partnerships with an HIV-infected female and HIV-uninfected male, vaginal insemination of a partner's semen during the fertile period coupled with $100 \%$ condom use may be the safest method of conception.

\section{Introduction}

It is estimated that there are 34 million HIV-infected people living worldwide with $68 \%$ residing in sub-Saharan Africa and $50 \%$ of cases occurring among women [1]. Serodiscordance, in which one person in a couple is HIV-infected and the other person is HIV-uninfected, is a common phenomenon. In a multisite collaborative study across East and Southern Africa, $49 \%$ of the enrolled heterosexual couples were HIV serodiscordant. HIV transmission within stable serodiscordant partnerships is thought to contribute substantially to the HIV epidemic in sub-Saharan Africa [2]. In the United States, it is estimated that there are more than 140,000 HIV serodiscordant heterosexual couples [3]. In HIV serodiscordant partnerships where conception occurs, the HIV-uninfected partner has a 1.8 (95\% confidence interval (CI) 1.01-3.26; $P<0.05)$ increased risk of HIV acquisition in comparison to partnerships where conception did not occur. The majority of HIVuninfected men and women in serodiscordant partnerships where conception occurs acquire HIV within the six months prior to conception and during the first six months of pregnancy indicating that couples engage in risky practices in order to conceive [4]. The per coital risk of HIV transmission from female-to-male is estimated at 0.0010 (95\% CI 0.00060-0.0017) in HIV serodiscordant couples [5].

In the United States, 52\% of HIV-infected women in a national probability study reported being in a serodiscordant partnership while $47 \%$ of HIV-infected women in subSaharan Africa are in stable serodiscordant relationships $[6,7]$. Evidence suggests that $20-50 \%$ of HIV-infected individuals desire children and this desire for childbearing may lead to unprotected sex and/or nondisclosure of HIV status, which in turn results in an increased risk of sexual HIV transmission $[4,6,8,9]$. In order to adequately curb HIV incidence, the reproductive desires and intentions of HIV serodiscordant couples must guide prevention interventions. Care for HIV-infected adults should include assessing reproductive goals in the context of the HIV status of one's sexual partners. Through the delivery of comprehensive reproductive healthcare, serodiscordant couples may fulfill their personal reproductive goals while decreasing the risk of sexual HIV transmission. 
To date, there is sparse information in the literature describing low-cost assisted reproductive methods, such as vaginal insemination, for HIV-infected females and HIVuninfected males (HIF/HUM) serodiscordant couples. In the following discussion, we will emphasize the use of vaginal insemination during the fertile period coupled with consistent condom use as a safer method of conception for HIF/HUM serodiscordant couples. The evaluation of safer methods of conception has been limited to the use of timed unprotected intercourse as well as sperm washing coupled with assisted reproductive procedures for HIV-infected males/HIV-uninfected female serodiscordant couples [10, $11]$.

\section{Ethical Implications}

It is time for society to normalize the lives of HIVinfected people including the basic human right to conceive and raise children $[12,13]$. All couples and individuals have the basic reproductive right "to decide freely and responsibly the number, spacing and timing of their children and to have the information and means to do so" [14]. Childbearing is important to many HIV-affected couples and healthcare providers have the responsibility of providing resources to help couples safely conceive while minimizing the risk of sexual and perinatal HIV transmission. In the current era where HIV-infected individuals are living longer, simply encouraging HIV-affected couples to abstain from procreation is no longer a realistic strategy, particularly in cultures where having children is stressed $[15,16]$. Inherent in this discussion is the conflict between the desire to have children and preventing HIV transmission [17]. Existing evidence suggests that HIV serodiscordant couples desiring conception seldom know of practices to reduce periconception sexual transmission and several knowingly risk HIV transmission to their partner in order to conceive [18].

\section{Childbearing Desires among HIV Serodiscordant Couples}

HIV serodiscordant couples may engage in risky sexual behaviors to conceive [4]. Various factors drive childbearing intentions amongst HIV serodiscordant couples, particularly, women wish to regain their self-status and pursue pregnancy as proof of recovery and evidence of regained self-control [9]. Societal and cultural expectations along with personal reproductive intentions may also drive HIVinfected women in serodiscordant relationships to conceive.

\section{Methods of Safer Conception}

Options for safer conception in HIF/HUM serodiscordant couples include antiretroviral therapy (ART), male circumcision, timed unprotected intercourse, preexposure prophylaxis (PrEP) for the uninfected male partner, assisted reproductive technology, and vaginal insemination of semen during the fertile period. The use of ART, timed unprotected intercourse, and assisted reproductive technology as safer methods of conception has been evaluated in HIF/HUM. The European studies evaluating the use of assisted reproductive technology in HIF/HUM serodiscordant couples illustrate that HIF may have underlying infertility or decreased response to ovarian stimulation as a result of HIV infection, ART, or tubal factor infertility $[19,20]$. Despite evidence supporting the protective effects of timed unprotected intercourse, PrEP for the uninfected partner, and assisted reproductive technology as safer methods of conception there are limited studies evaluating the acceptability and feasibility of these methods amongst HIV serodiscordant couples.

4.1. Antiretroviral Therapy (ART). Prior to initiation of conception attempts, other risk reduction interventions should be considered to reduce HIV transmission to the HUM. Ideally, the HIF should be on ART and attain an undetectable plasma viral load. While fully suppressive ART use by the HIV-infected individual dramatically reduces the chance of sexual transmission, sexual HIV transmission may still occur [21]. A systematic review evaluating the rate of HIV transmission through unprotected intercourse in serodiscordant couples with the HIV-infected partner on ART noted an overall transmission rate of 0.46 (95\% CI 0.19-1.09) per 100 person years [22]. Early initiation of ART is associated with a 96\% (95\% CI 0.01-0.27) relative reduction of HIV transmission to the HIV-negative partner [23]. In the United States, ART is recommended for all HIV-infected individuals [24]. However, initiation of ART with an undetectable serum HIV RNA viral load does not ensure an undetectable viral load in the genital tract. Therefore, couples should be counseled that suppressed plasma viremia does not guarantee that an individual is sexually noninfectious [25-28].

4.2. Male Circumcision. Reduction of HIV transmission to the male partner can also be assisted with male circumcision. Male circumcision, the surgical removal of all or part of the foreskin of the penis, is thought to remove a potential site of entry for HIV infection [29]. Circumcision can reduce the risk of HIV transmission to the index HIV-uninfected male partner by $38-66 \%$ over 24 months, assuming an adequate duration of healing prior to reinitiation of sexual activity [30-32]. Circumcision coupled with other safer methods of conception may decrease the risk of sexual HIV transmission.

4.3. Timed Unprotected Intercourse. Natural conception for HIV serodiscordant couples desiring conception involves timing unprotected sexual intercourse during the fertile period. Women are taught to monitor their menstrual cycle to determine the day of ovulation using the basal body temperature, calendar calculation, ovulation prediction kits assessing urinary hormones, and/or monitoring of their cervical mucus. Couples are encouraged to minimize their sexual encounters to the fertile period to decrease the number of unprotected sexual encounters while optimizing their chance of conception. In Spain, there was a cohort of $62 \mathrm{HIV}$ serodiscordant couples with $22 \mathrm{HIF} / \mathrm{HUM}$ where timed unprotected intercourse occurred and the female was 
receiving suppressive ART; there were no cases of sexual HIV transmission [33]. On the other hand, in France, Mandelbrot et al. found a $4 \%$ risk of male-to-female HIV transmission among 92 HIV serodiscordant couples counseled on the use of timed unprotected intercourse. All of the cases of HIV transmission occurred during unprotected sex outside the fertile period and only 21 of the HIV-infected men were receiving ART [11]. Nonetheless, these cases highlight that couples may not adhere to unprotected intercourse only during the fertile period.

4.4. Periconception Preexposure Prophylaxis (PrEP) or "PrEPception". PrEP for the HIV-negative partner prior to attempted conception, "PrEPception," combined with timed unprotected intercourse is another risk reduction technique that may minimize the risk of sexual HIV transmission among serodiscordant couples [3]. In Switzerland, a cohort of 53 HIV serodiscordant couples (male positive, female negative) desiring conception, 46 couples opted for PrEP 12 and 36 hours prior to timed unprotected intercourse and there were no cases of HIV transmission. After the first attempt with timed unprotected intercourse, the pregnancy rate was $26 \%$ which increased to $66 \%$ after five attempts and reached a plateau of $75 \%$ after 12 attempts. PrEP coupled with time unprotected intercourse may help at risk individuals reduce their risk of HIV acquisition during conception [34].

The efficacy of preexposure prophylaxis (PrEP) in HIV serodiscordant couples has been demonstrated, yet its widespread use may be restricted because of issues related to adherence, adequate regimens, cost effectiveness, and use in resource-limited environments [35-41]. PrEP should be available for serodiscordant couples as a risk reduction strategy to prevent sexual HIV transmission. Clinical trials to date have not tested the use of PrEP on an other than daily basis with the exception of the evaluation of tenofovir gel, which is not commercially available, and oral PrEP in HIV serodiscordant couples desiring conception in Switzerland $[34,36,37]$. The data on PrEP from these trials is mixed. The United States Food and Drug Administration has endorsed tenofovir/emtricitabine (Truvada) for use as once daily PrEP in men and women at high risk of sexually acquired HIV infection.

4.5. Assisted Reproductive Technology. Comprehensive reproductive counseling and assisted reproductive technology services are limited for HIV-infected women in serodiscordant relationships desiring pregnancy in resource-limited environments. In developed countries, assisted reproductive technology is not readily available and may be cost prohibitive to couples. In the United States, it has been reported that less than $5 \%$ of at least 400 assisted reproductive clinics provide services to HIV-infected individuals [42]. In HIF/HUM serodiscordant couples laborious and expensive reproductive techniques may not be necessary to reduce the risk of HIV transmission during attempted conception, unless there is a documented history of infertility [43]. HIVinfected women may have decreased reproductive potential in terms of ovarian response to stimulation, fertilization, and implantation [44]. In-vitro fertilization with intracytoplasmic intrauterine insemination may improve the rates of fertilization in HIV-infected females with known infertility.

4.6. Vaginal Insemination. Vaginal insemination along with consistent male condom use in a heterosexual HIF/HUM serodiscordant partnership during the fertile period may be the easiest and safest reproductive option to further reduce the risk of sexual HIV transmission compared to timed unprotected sexual intercourse. Vaginal insemination can be performed at home by the HIF/HUM serodiscordant couple during the fertile period of the menstrual cycle after collection of semen from a water-based lubricated condom or clean container.

\section{Vaginal Insemination as the Safer Conception Option for HIF/HUM Serodiscordant Couples}

The use of vaginal insemination as a safer method of conception may help combat the sexual transmission of HIV [45]. Nonetheless, there are no data in the literature reporting on vaginal insemination despite anecdotal reports supporting its use. To date, the only study registered with ClinicalTrials.gov evaluating the acceptability, feasibility, and efficacy of vaginal insemination amongst HIV serodiscordant couples (HIF/HUM) desiring conception will be conducted in Kisumu, Kenya (ClinicalTrials.gov identifier: NCT01468753).

Vaginal insemination involves the woman monitoring her menstrual cycle to estimate her most fertile days. Semen is collected either during sex with a nonspermicidal (waterbased lubricated) condom or via ejaculation into a clean container (Figure 1). The semen is then aspirated shortly after ejaculation into a needless syringe or a bulb pipette. The woman lies on her back with her hips elevated while the semen is slowly deposited into the vagina by herself or her partner.

Despite the theoretical ease of the vaginal insemination procedures, there are potential challenges that may impede its acceptability and efficacy. Determination of the fertile days in the menstrual cycle without advance technologies in a resource-limited setting may prove difficult. Without accurate determination of the fertile days in an ovulating woman, the vaginal insemination procedures may be ineffective, unless couples correctly time vaginal insemination for use during the fertile period. There are five established methods of predicting ovulation: serial ultrasound, calendar calculation, basal body temperature, consistency of cervical mucus, and urine hormones. Ultrasound detection of the day of ovulation is a direct method and most accurate but is unavailable outside of a clinical facility. Calendar calculation and basal body temperature are considered to be unreliable, however, thin, clear, stretchy cervical mucus (spinnbarkeit) and urine luteinizing hormone are the two methods that correlate best with ultrasound detection of ovulation [46, 47]. In addition to the challenge of determining the fertile period of the cycle, 


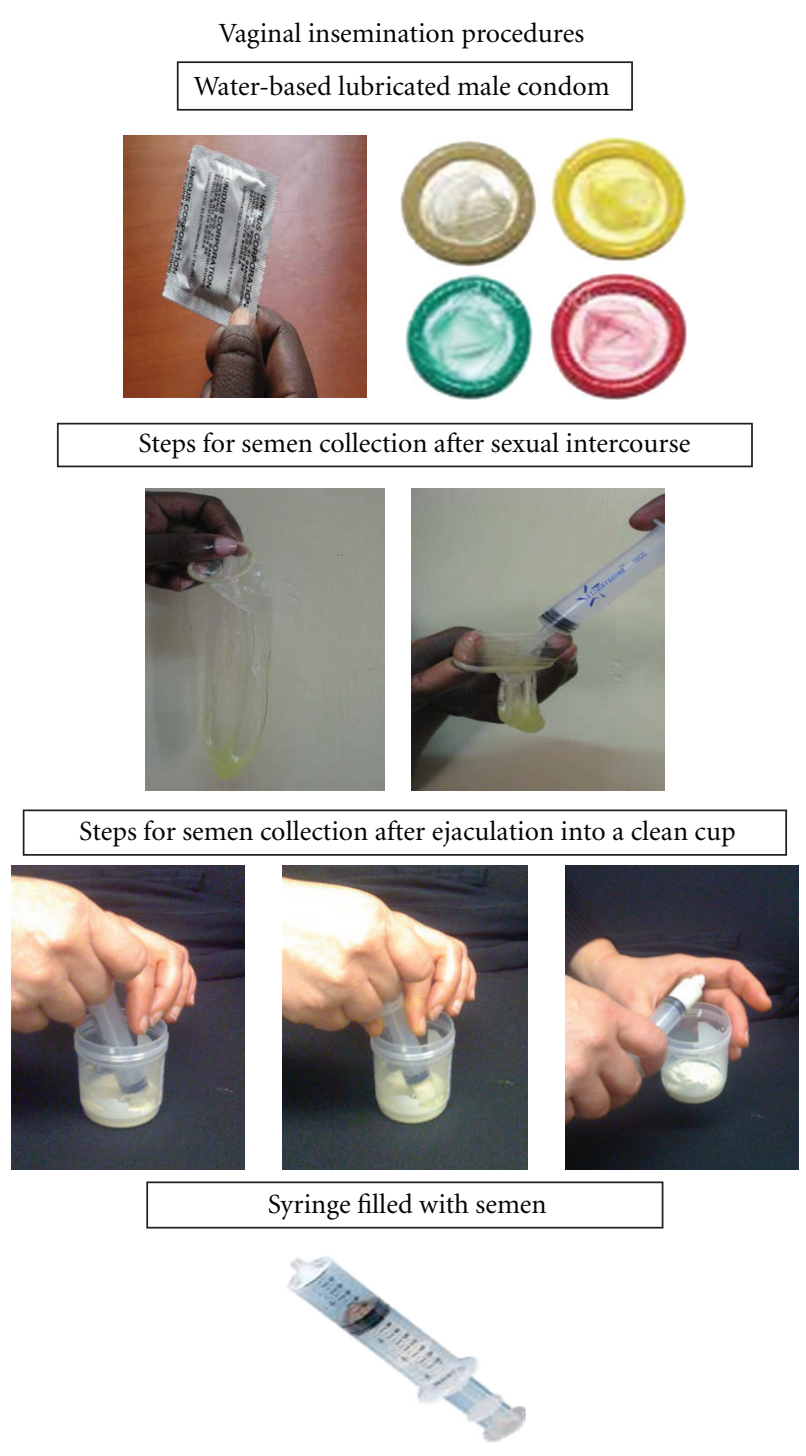

FIGURE 1: Steps for vaginal insemination of semen: (1) on a fertile day of the menstrual cycle, engage in sex using nonspermicidal condom (water-based lubricated or nonlubricated condom) or semen can be collected from a clean cup after ejaculation. (2) Collect the semen with a plastic needle-less syringe from a condom or clean cup. (3) The female or male partner will insert the syringe with the semen as high as possible into the vagina, ideally immediately after ejaculation. (4) The female partner may lie down for at least thirty minutes after insertion. (5) Steps (1-4) may be repeated every other day during the fertile period.

couples may also encounter cultural and technical challenges with performing the vaginal insemination. Couples may be uncomfortable with the insemination process, use of the devices, or touching their genitalia to perform the vaginal insemination procedures. The incorporation of continued counseling and education may help couples overcome these potential challenges as they prepare for safer conception with vaginal insemination [48]. The interest and motivation amongst HIV serodiscordant couples may also contribute to overcoming the perceived cultural and technical barriers [49].

\section{Guidelines for Healthcare Providers}

In HIF/HUM serodiscordant couples, there are three unique clinical challenges: maintaining the woman's health before, during, and after the pregnancy; preventing perinatal transmission; preventing sexual transmission to the HIV negative partner [16]. In order to facilitate conception and a safe pregnancy, the health of both members of the couple should be optimized. The HIV-infected woman should have a thorough clinical assessment (CD4 count, viral load, and genotype if available), evaluation of antiretroviral regimen, identification and management of comorbidities such as tuberculosis, diabetes, or hypertension in addition to other transmissible infections or cofactors that may increase the risk of HIV transmission or acquisition [10, 12]. Similarly, an HIV-infected person should be counselled on the risks of conception with a detectable HIV-1 RNA viral load, documented infertility or presence of conditions affecting fertility in either partner, nondisclosure of HIV status, or medical contraindications to pregnancy [12]. In couples with documented infertility, they may be referred to a fertility clinic for preconception counseling and discussion of available options, if available. If either partner has conditions affecting fertility the couple can be allowed to continue their attempts at conception; however, they should be encouraged to seek assisted reproductive technology services after six unsuccessful attempts at vaginal insemination or timed unprotected intercourse over six cycles [12, 34]. Couples should also have a clinical assessment to screen and treat for sexually transmitted infections prior to attempts at conception [12]. In eligible HIV-infected women, antiretroviral therapy (ART) should be initiated and optimized to improve their health status and reduce the risk of HIV transmission [23]. The use of combination antiretroviral regimens in HIVinfected women is associated with a near elimination of perinatal HIV transmission [50].

Male circumcision, ART, timed unprotected intercourse, PrEP for the uninfected male partner, and vaginal insemination of semen during the fertile period can be used in concert for HIF/HUM desiring conception. For example, an HIF on ART may attempt timed unprotected intercourse or vaginal insemination with her HUM partner that has been circumcised. Together, these low-cost interventions may allow HIF/HUM serodiscordant couples to safely conceive while decreasing the risk of sexual HIV transmission.

Preventing sexual transmission of HIV to the uninfected partner involves counseling on the consistent use of condoms with all sexual encounters while attempting to conceive with timed unprotected intercourse, PrEP, assisted reproductive technology, or vaginal insemination. Despite these recommendations, the barriers to consistent condom use should not be overlooked. Difficulties that have been reported with consistent condom use include loss of spontaneity, reduced libido, and decreased frequency of sexual intercourse [17]. However, healthcare providers can help HIV serodiscordant couples overcome these challenges with an emphasis on their motivation to safely conceive and minimize sexual HIV transmission. It has been argued that couples and medical providers accepting the risk of transmitting a disease to their 
offspring do not act unethically if all reasonable precautions to prevent transmission are taken $[33,51]$.

As the paradigm shifts to assisting HIV-affected couples fulfill their reproductive goals of procreating, healthcare providers must also begin to expand their understanding of the family planning concept beyond contraception to include methods of safer conception. This more comprehensive concept of reproductive healthcare also includes safer methods of conception. The integration of comprehensive reproductive healthcare services into HIV care and treatment programs will strengthen the repertoire of medical services available to HIV-affected couples desiring children.

\section{Future Directions}

The armamentarium of safer methods of conception for HIV serodiscordant couples desiring conception is expanding. Existing evidence supports the use of ART, male circumcision, timed unprotected intercourse, PrEP, and assisted reproductive technology as safer methods of conception for serodiscordant couples. Although the existing evidence may have some limitations, they can be used alone or in combination to decrease the risk of sexual HIV transmission while attempting conception. Anecdotal evidence supports the use of vaginal insemination as a low-cost and feasible method of safer conception by HIF/HUM serodiscordant couples. However, the paucity of existing data supporting the use of vaginal insemination calls for studies to address the feasibility and efficacy of this method. We anticipate that the current study evaluating vaginal insemination in a low resource environment will address the perceived cultural and technical challenges with performing the vaginal insemination procedures in a monogamous HIF/HUM serodiscordant partnership. The findings of this study will assist in establishing guidelines for its use as a component of comprehensive reproductive healthcare for HIV-affected couples. In the future, we expect that periovulatory vaginal insemination coupled with consistent condom use will be widely supported and endorsed by healthcare providers for HIF/HUM serodiscordant partnerships.

\section{Conclusion}

Improvements in HIV care and treatment allow HIVinfected individuals around the world to live longer and consider their reproductive goals. In an effort to help $\mathrm{HIV}$-infected individuals meet these goals, HIV serodiscordant couples need access to methods of safer conception. Although there have been great gains with the advent of reproductive technologies to assist with conception, unfortunately these technologies are not readily available for most people affected by HIV and fortunately, not necessary for a large majority of HIV serodiscordant couples with preserved fertility. Periovulatory vaginal insemination coupled with consistent condom use may allow HIF/HUM serodiscordant couples to fulfill their desire of having children without risking sexual HIV transmission.

\section{References}

[1] UNAIDS World AIDS Day Report, 2011, http://www.unaids .org/en/media/unaids/contentassets/documents/unaidspublication/2011/JC2216_WorldAIDSday_report_2011_en.pdf.

[2] J. R. Lingappa, B. Lambdin, E. A. Bukusi et al., "Regional differences in prevalence of HIV-1 discordance in Africa and enrollment of HIV-1 discordant couples into an HIV-1 prevention trial," PLoS ONE, vol. 3, no. 1, Article ID e1411, 2008.

[3] M. A. Lampe, D. K. Smith, G. J. E. Anderson, A. E. Edwards, and S. R. Nesheim, "Achieving safe conception in HIVdiscordant couples: the potential role of oral preexposure prophylaxis (PrEP) in the United States," American Journal of Obstetrics and Gynecology, vol. 204, no. 6, pp. 488.e1-488.e8, 2011.

[4] S. G. Brubaker, E. A. Bukusi, J. Odoyo, J. Achando, A. Okumu, and C. R. Cohen, "Pregnancy and HIV transmission among HIV-discordant couples in a clinical trial in Kisumu, Kenya," HIV Medicine, vol. 12, no. 5, pp. 316-321, 2011.

[5] J. P. Hughes, J. M. Baeten, J. R. Lingappa et al., "Determinants of per-coital act HIV-1 infectivity among African HIV-1 serodiscordant couples," Journal of Infectious Disease, vol. 205, no. 3, pp. 358-365, 2012.

[6] J. L. Chen, K. A. Phillips, D. E. Kanouse, R. L. Collins, and A. Miu, "Fertility desires and intentions of HIV-positive men and women," Family Planning Perspectives, vol. 33, no. 4, pp. 144-152, 2001.

[7] O. Eyawo, D. de Walque, N. Ford, G. Gakii, R. T. Lester, and E. J. Mills, "HIV status in discordant couples in sub-Saharan Africa: a systematic review and meta-analysis," The Lancet Infectious Diseases, vol. 10, no. 11, pp. 770-777, 2010.

[8] L. T. Matthews, J. M. Baeten, C. Celum, and D. R. Bangsberg, "Periconception pre-exposure prophylaxis to prevent HIV transmission: benefits, risks, and challenges to implementation," AIDS, vol. 24, no. 13, pp. 1975-1982, 2010.

[9] O. Awiti Ujiji, A. M. Ekström, F. Ilako, D. Indalo, and B. Rubenson, "I will not let my HIV status stand in the way'. Decisions on motherhood among women on ART in a slum in Kenya-a qualitative study," BMC Women's Health, vol. 10, article 13, 2010.

[10] P. Barreiro, J. del Romero, M. Leal et al., "Natural pregnancies in HIV-serodiscordant couples receiving successful antiretroviral therapy," Journal of Acquired Immune Deficiency Syndromes, vol. 43, no. 3, pp. 324-326, 2006.

[11] L. Mandelbrot, I. Heard, E. Henrion-Géant, and R. Henrion, "Natural conception in HIV-negative women with HIVinfected partners," The Lancet, vol. 349, no. 9055, pp. 850-851, 1997.

[12] L. G. Bekker, V. Black, L. Myer et al., "Guideline on safer conception in fertile HIV-infected individuals and couples," Southern African Journal of HIV Medicine, vol. 12, no. 3, pp. 31-44, 2011.

[13] S. Gruskin, R. Firestone, S. MacCarthy, and L. Ferguson, "HIV and pregnancy intentions: do services adequately respond to women's needs?" American Journal of Public Health, vol. 98, no. 10 , pp. 1746-1750, 2008.

[14] WHO Definition of Reproductive Rights and United Nations, "Programme of action," in International Conference on Population Development, Cairo, September 1994, Paragraph 7.12.

[15] A. van Sighem, L. Gras, P. Reiss, K. Brinkman, and F. de Wolf, "Life expectancy of recently diagnosed asymptomatic HIVinfected patients approaches that of uninfected individuals," AIDS, vol. 24, no. 10, pp. 1527-1535, 2010. 
[16] L. T. Matthews and J. S. Mukherjee, "Strategies for harm reduction among HIV-affected couples who want to conceive," AIDS and Behavior, vol. 13, supplement 1, pp. S5-S11, 2009.

[17] L. C. Rispel, C. A. Metcalf, K. Moody, A. Cloete, and G. Caswell, "Sexual relations and childbearing decisions of HIVdiscordant couples: an exploratory study in South Africa and Tanzania," Reproductive Health Matters, vol. 19, no. 37, pp. 184-193, 2011.

[18] L. T. Matthews, T. Crankshaw, J. Giddy et al., "Reproductive decision-making and periconception practices among HIVpositive men and women accessing HIV care in Durban," in Proceedings of the 6th IAS Conference on HIV Pathogenesis, Treatment and Prevention, Rome, Italy, 2011, Poster Exhibition TUPE325.

[19] O. Coll, A. Suy, F. Figueras et al., "Decreased pregnancy rate after in-vitro fertilization in HIV-infected women receiving HAART," AIDS, vol. 20, no. 1, pp. 121-123, 2006.

[20] P. Terriou, P. Auquier, V. Chabert-Orsini et al., "Outcome of ICSI in HIV-1-infected women," Human Reproduction, vol. 20, no. 10, pp. 2838-2843, 2005.

[21] M. Stürmer, H. W. Doerr, A. Berger, and P. Gute, "Is transmission of HIV-1 in non-viraemic serodiscordant couples possible?" Antiviral Therapy, vol. 13, no. 5, pp. 729-732, 2008.

[22] S. Attia, M. Egger, M. Müller, M. Zwahlen, and N. Low, "Sexual transmission of HIV according to viral load and antiretroviral therapy: systematic review and meta-analysis," AIDS, vol. 23, no. 11, pp. 1397-1404, 2009.

[23] M. S. Cohen, Y. Q. Chen, M. McCauley et al., "Prevention of HIV-1 infection with early antiretroviral therapy," The New England Journal of Medicine, vol. 365, no. 6, pp. 493-505, 2011.

[24] Adult ART Guidelines, June 2012, Panel on Antiretroviral Guidelines for Adults and Adolescents. Guidelines for the use of antiretroviral agents in HIV-1-infected adults and adolescents. Department of Health and Human Services, Initiating Antiretroviral Therapy in Treatment-Naïve Patients, http://aidsinfo.nih.gov/contentfiles/lvguidelines/AdultandAdolescentGL.pdf.

[25] G. Lorello, C. la Porte, R. Pilon, G. Zhang, T. Karnauchow, and P. MacPherson, "Discordance in HIV-1 viral loads and antiretroviral drug concentrations comparing semen and blood plasma," HIV Medicine, vol. 10, no. 9, pp. 548-554, 2009.

[26] J. D. Homans, S. Christensen, C. H. Wang et al., "Permissive and protective factors associated with presence, level and longitudinal pattern of cervicovaginal HIV shedding," Journal of Acquired Deficiency Syndrome, vol. 60, no. 1, pp. 99-110, 2012.

[27] G. Liuzzi, A. Chirianni, M. Clementi et al., "Analysis of HIV-1 load in blood, semen and saliva: evidence for different viral compartments in a cross-sectional and longitudinal study," AIDS, vol. 10, no. 14, pp. F51-F56, 1996.

[28] P. L. Vernazza, L. Troiani, M. J. Flepp et al., "Potent antiretroviral treatment of HIV-infection results in suppression of the seminal shedding of HIV," AIDS, vol. 14, no. 2, pp. 117-121, 2000.

[29] N. Siegfried, M. Muller, J. J. Deeks, and J. Volmink, "Male circumcision for prevention of heterosexual acquisition of HIV in men," Cochrane Database of Systematic Reviews, no. 2, Article ID CD003362, 2009.

[30] B. Auvert, D. Taljaard, E. Lagarde, J. Sobngwi-Tambekou, R. Sitta, and A. Puren, "Randomized, controlled intervention trial of male circumcision for reduction of HIV infection risk: the ANRS 1265 trial," PLoS Medicine, vol. 2, no. 11, article e298, 2005.
[31] R. C. Bailey, S. Moses, C. B. Parker et al., "Male circumcision for HIV prevention in young men in Kisumu, Kenya: a randomised controlled trial," The Lancet, vol. 369, no. 9562, pp. 643-656, 2007.

[32] R. H. Gray, G. Kigozi, D. Serwadda et al., "Male circumcision for HIV prevention in men in Rakai, Uganda: a randomised trial," The Lancet, vol. 369, no. 9562, pp. 657-666, 2007.

[33] P. Barreiro, A. Duerr, K. Beckerman, and V. Soriano, "Reproductive options for HIV-serodiscordant couples," AIDS Reviews, vol. 8, no. 3, pp. 158-170, 2006.

[34] P. L. Vernazza, I. Graf, U. Sonnenberg-Schwan, M. Geit, and A. Meurer, "Pre-exposure prophylaxis and timed intercourse for HIV-discordant couples willing to conceive a child," AIDS, vol. 25, no. 16, pp. 2005-2008, 2011.

[35] Pivotal Study Finds That HIV Medications are Highly Effective as Prophylaxis against HIV Infection in Men and Women in Africa, University of Washington International Clinical Research Center, Washington, DC, USA, 2012.

[36] R. M. Grant, J. R. Lama, P. L. Anderdson et al., "Preexposure chemoprophylaxis for HIV prevention in men who have sex with men," The New England Journal of Medicine, vol. 363, no. 27, pp. 2587-2599, 2010.

[37] Q. A. Karim, S. S. A. Karim, J. A. Frohlich et al., "Effectiveness and safety of tenofovir gel, an antiretroviral microbicide, for the prevention of HIV infection in women," Science, vol. 329, no. 5996, pp. 1168-1174, 2010.

[38] T. Kelesidis and R. J. Landovitz, "Preexposure prophylaxis for HIV prevention," Current HIV/AIDS Reports, vol. 8, no. 2, pp. 94-103, 2011.

[39] M. S. Cohen, K. E. Muessig, M. K. Smith et al., "Antiviral agents and HIV prevention: controversies, conflicts and consensus ," AIDS. In press.

[40] R. P. Walensky, J. E. Park, R. Wood et al., "The costeffectiveness of pre-exposure prophylaxis for HIV infection in South African women," Clinical Infectious Disease, vol. 54, no. 10, pp. 1504-1513, 2012.

[41] T. R. Koppenhaver, W. S. Sorenson, W. Stephen et al., "The cost-effectiveness of pre-exposure prophylaxis in men who have sex with men in the United States: an epidemic model," Journal of Acquired Immune Deficiency Syndromes, vol. 58, no. 2, pp. e51-e52, 2011.

[42] E. S. Daar and J. F. Daar, "Human immunodeficiency virus and fertility care: embarking on a path of knowledge and access," Fertility and Sterility, vol. 85, no. 2, pp. 298-300, 2006.

[43] J. Ohl, M. Partisani, C. Wittemer et al., "Assisted reproduction techniques for HIV serodiscordant couples: 18 months of experience," Human Reproduction, vol. 18, no. 6, pp. 12441249, 2003.

[44] A. E. Semprini, L. H. Hollander, A. Vucetich, and C. GillingSmith, "Infertility treatment for HIV-positive women," Women's Health, vol. 4, no. 4, pp. 369-382, 2008.

[45] Recommendations for Use of Antiretroviral Drugs in Pregnant HIV-1 Infected Women for Maternal Health and Interventions to Reduce Perinatal HIV Transmission in the United States. Section: Reproductive Options for HIV Concordant and Serodiscordant Couples, http://www.aidsinfo.nih.gov/guidelines/html/3/perinatal-guidelines/153/reproductive-options-for-hiv-concordant-and-serodiscordantcouples, 2011.

[46] R. Ecochard, H. Boehringer, M. Rabilloud, and H. Marret, "Chronological aspects of ultrasonic, hormonal, and other indirect indices of ovulation," British Journal of Obstetrics and Gynaecology, vol. 108, no. 8, pp. 822-829, 2001. 
[47] A. C. Thornton, F. Romanelli, and J. D. Collins, "Reproduction decision making for couples affected by HIV: a review of the literature," Topics in HIV Medicine, vol. 12, no. 2, pp. 61-67, 2004.

[48] O. Mmeje, M. Workneh, B. Njoroge et al., "Perspectives on vaginal insemination as a safer method of conception among HIV serodiscordant couples and their healthcare providers in Kisumu, Kenya," AIDS Research and Treatment. In press.

[49] S. G. Brubaker, L. Darbes, E. A. Bukusi et al., "Risk perception and the desire for children among HIV discordant couples in Kisumu, Kenya," in Proceedings of the 6th IAS Conference on HIV Pathogenesis and Treatment, International AIDS Society, 2010, TUPE 332.

[50] R. Tubiana, S. Matheron, J. LeChenadec et al., "Extremely low risk of HIV in women starting HAART before pregnancy: french perinatal cohort, ANRS EPF CO1/11," in Proceedings of the 18th Conference on Retroviruses and Opportunistic Infections, 2011, 735.

[51] The Ethics Committee of the American Society for Reproductive Medicine, "Human immunodeficiency virus and infertility treatment," Fertility and Sterility, vol. 82, supplement 1, pp. S228-S231, 2004. 


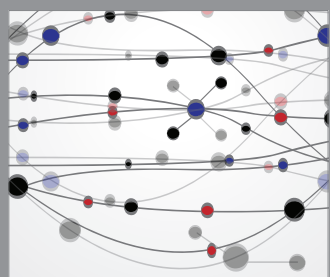

The Scientific World Journal
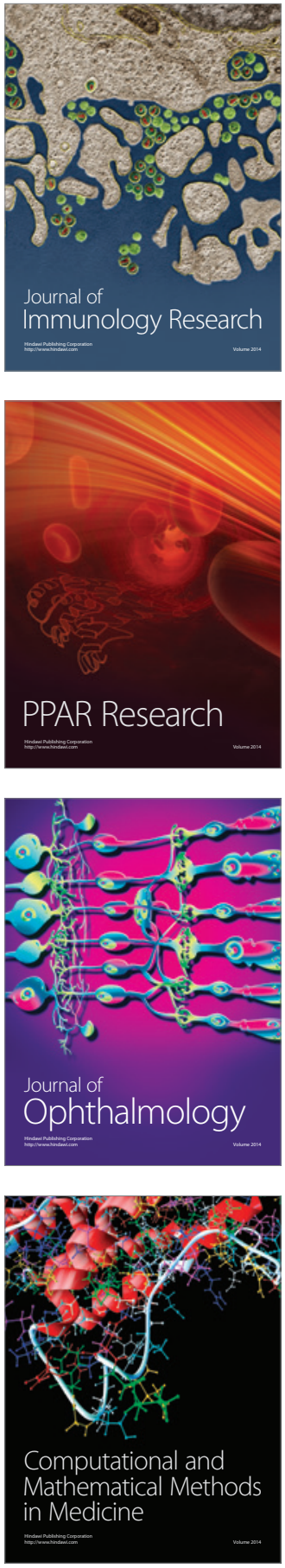

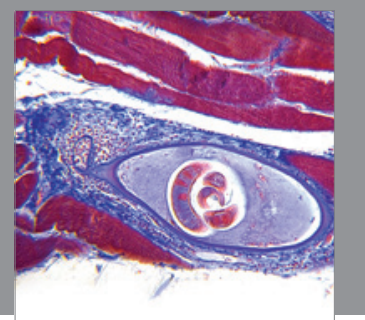

Gastroenterology

Research and Practice
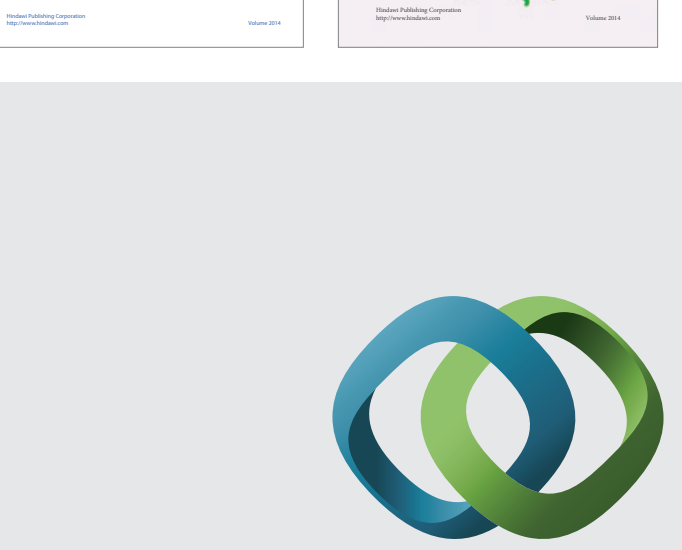

\section{Hindawi}

Submit your manuscripts at

http://www.hindawi.com
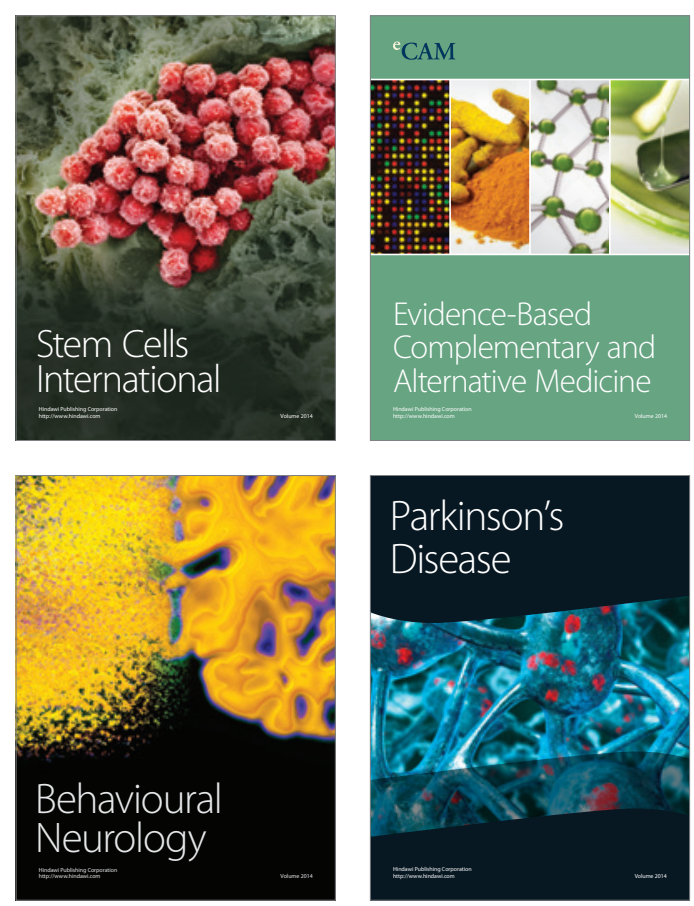

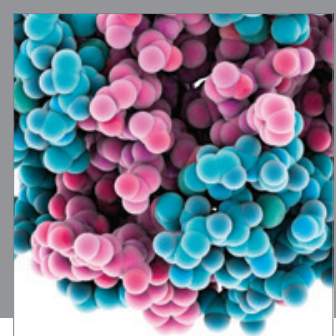

Journal of
Diabetes Research

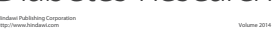

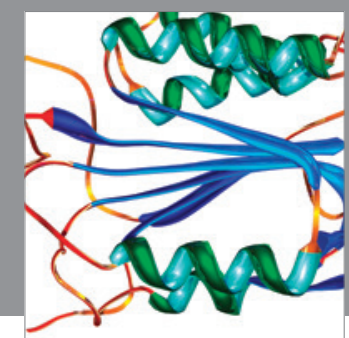

Disease Markers
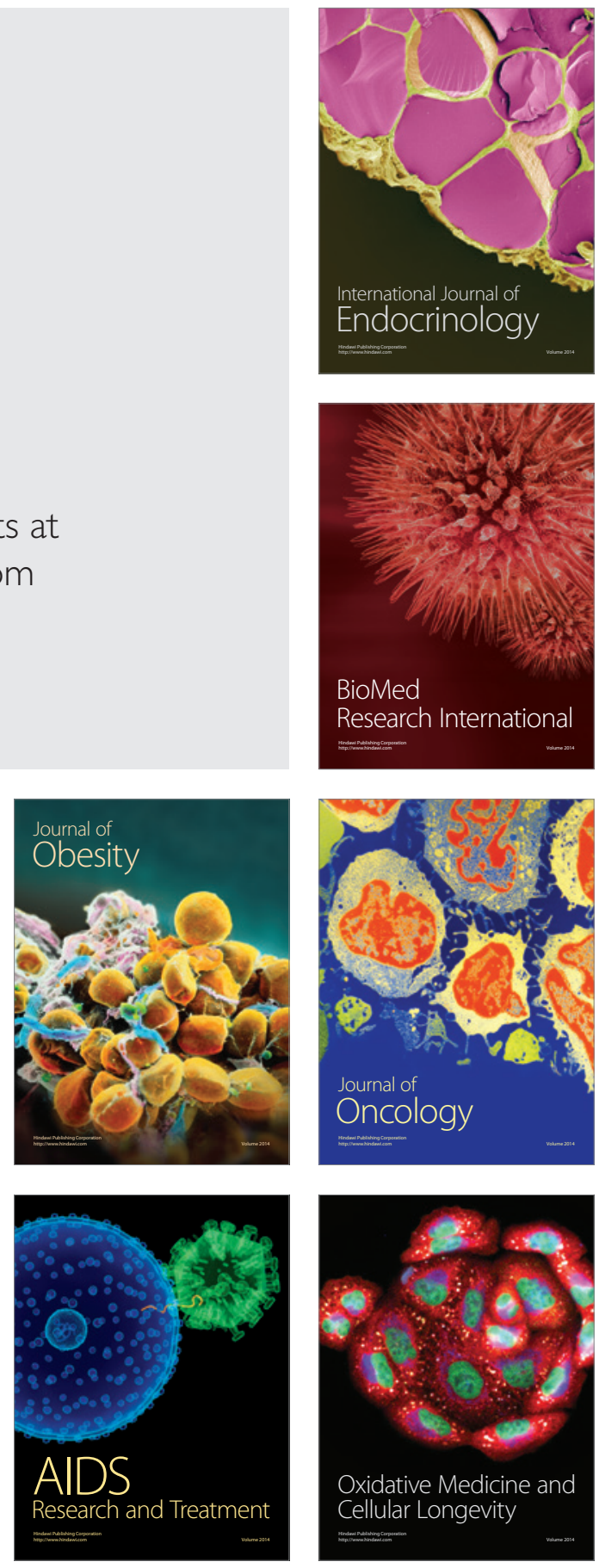